\title{
BAYESIAN QUICKEST DETECTION PROBLEMS FOR SOME DIFFUSION PROCESSES
}

\author{
PAVEL V. GAPEEV, ${ }^{*}$ London School of Economics \\ ALBERT N. SHIRYAEV, ${ }^{* *}$ Steklov Institute of Mathematics
}

\begin{abstract}
We study the Bayesian problems of detecting a change in the drift rate of an observable diffusion process with linear and exponential penalty costs for a detection delay. The optimal times of alarms are found as the first times at which the weighted likelihood ratios hit stochastic boundaries depending on the current observations. The proof is based on the reduction of the initial problems into appropriate three-dimensional optimal stopping problems and the analysis of the associated parabolic-type free-boundary problems. We provide closed-form estimates for the value functions and the boundaries, under certain nontrivial relations between the coefficients of the observable diffusion.
\end{abstract}

Keywords: Disorder detection; diffusion process; multidimensional optimal stopping; stochastic boundary; a change-of-variable formula with local time on surfaces; parabolictype free-boundary problem

2010 Mathematics Subject Classification: Primary 60G40; 62M20; 34K10

Secondary $62 \mathrm{C} 10 ; 62 \mathrm{~L} 15 ; 60 \mathrm{~J} 60$

\section{Introduction}

The problem of quickest disorder detection for an observable diffusion process seeks to determine a stopping time of alarm $\tau$ which is as close as possible to the unknown time of disorder (or change point) $\theta$ at which the local drift rate of the process changes from $\mu_{0}(\cdot)$ to $\mu_{1}(\cdot)$. In the classical Bayesian formulation, it is assumed that the random time $\theta$ takes the value 0 with probability $\pi$ and is exponentially distributed with parameter $\lambda>0$ given that $\theta>0$. An optimality criterion was proposed in [23] and [24] for the time of alarm to minimize a linear combination of the false alarm probability and the expected time delay in detecting the disorder correctly, for sequences of independent and identically distributed observations. An explicit solution of the problem of detecting a change in the constant drift rate of an observable Wiener process with the same optimality criterion was derived in [26] and [27]. The appropriate optimal stopping problem for the posterior probability of the occurrence of disorder was reduced to the associated free-boundary problem for an ordinary differential operator (see also [28, Chapter IV, Section 4] or [18, Chapter VI, Section 22]). A finite time horizon version of the Wiener disorder problem was studied in [10].

The idea of replacing the initial average time delay by a certain nonadditive detection delay penalty criterion was originally introduced in [25]. The resulting Bayesian risk function was

Received 7 November 2011; revision received 21 June 2012.

* Postal address: Department of Mathematics, London School of Economics, Houghton Street, London, WC2A 2AE, UK. Email address: p.v.gapeev@1se.ac.uk

Supported by the Alexander von Humboldt Fellowship for Experienced Researchers.

** Postal address: Steklov Institute of Mathematics, Russian Academy of Sciences, Gubkina Street 8, Moscow 119991, Russia. Email address: albertsh@mi.ras.ru 
expressed through the current state of a multidimensional Markovian sufficient statistic, having state space components which are different from the posterior probability. Such a process contained all the necessary information to determine the structure of the optimal time of alarm (see also the more recent works [30], [31], and [6]). In the case of exponential penalty costs for a delay, it was observed by Poor [19] that the weighted likelihood ratio process turns out to be a one-dimensional Markovian sufficient statistic, for sequences of independent and identically distributed observations. This idea was developed further by Beibel [4], who solved the corresponding problem of detecting a change in the drift rate of an observable Wiener process as a generalized parking problem. Bayraktar and Dayanik [1] recognized the same property from the structure of the ordinary differential-difference equation in the free-boundary problem associated with the Bayesian problem of detecting a change in the constant intensity rate of an observable Poisson process. Some other formulations of the problem for the case of detecting a change in the arrival rate of a Poisson process, leading to the appearance of essentially multidimensional Markovian sufficient statistics, were studied by Bayraktar, Dayanik, and Karatzas in [2] and [3]. Extensive overviews of these and other related quickest sequential change-point detection methods were provided in the monographs [29] and [20].

In the present paper we study the Bayesian quickest disorder detection problems for observable diffusions with linear and exponential delay penalty costs. We reduce the initial problems to extended optimal stopping problems for three-dimensional Markov diffusion processes, having the posterior probability, weighted likelihood ratio, and the observations as their state space components. We show that the optimal stopping times are expressed as the first times at which the weighted likelihood ratio processes hit stochastic boundaries depending on the current state of the observation process only. We verify that the value functions and the optimal stopping boundaries are characterized by means of the associated free-boundary problem for a second-order partial differential operator. The latter turns out to be of parabolic type, because the observation process is a one-dimensional diffusion. We also derive closedform estimates for the value functions and the boundaries for a special nontrivial subclass of observable diffusions. The Bayesian sequential testing problem for such processes was recently solved in [11]. Another related problem of transient signal detection and identification of twosided changes in the drift rates of observable diffusion processes was considered by Pospisil, Vecer and Hadjiliadis in [21].

The paper is organized as follows. In Section 2 we formulate the Bayesian quickest disorder detection problem for observable diffusion processes with linear and exponential delay penalty costs, and construct the appropriate multidimensional optimal stopping problem. In Sections 3 and 4, we present the associated free-boundary problem and reduce the resulting parabolic-type partial differential operator to the normal form, which is amenable for further considerations. Applying the change-of-variable formula with local time on surfaces, obtained by Peskir [17], we verify that the solution of the free-boundary problem, which satisfies certain additional conditions, provides the solution of the initial optimal stopping problem. We derive closed-form estimates for the value function and the boundary, which are uniquely determined as solutions of ordinary differential equations, under certain nontrivial relations between the coefficients of the observable diffusion. The main results are stated in Theorems 3.1 and 4.1.

\section{Preliminaries}

In this section we give the Bayesian formulation of the problem (see [28, Chapter IV, Section 4] or [18, Chapter VI, Section 22] for the case of Wiener processes) in which it is assumed that one observes a sample path of the diffusion process $X=\left(X_{t}\right)_{t \geq 0}$ with the drift 
rate changing from $\mu_{0}(\cdot)$ to $\mu_{1}(\cdot)$ at some random time $\theta$ taking the value 0 with probability $\pi$ and being exponentially distributed with parameter $\lambda>0$ under $\theta>0$.

\subsection{Formulation of the problem}

Suppose that, on a probability space $\left(\Omega, \mathcal{F}, \mathbb{P}_{\pi}\right)$, there exists a standard Brownian motion $B=\left(B_{t}\right)_{t \geq 0}$, being independent of a nonnegative random variable $\theta$ such that $\mathbb{P}_{\pi}(\theta=0)=\pi$ and $\mathbb{P}_{\pi}(\theta>t \mid \theta>0)=\mathrm{e}^{-\lambda t}$ for all $t \geq 0$ and some fixed $\lambda>0$. Let $X=\left(X_{t}\right)_{t \geq 0}$ be a continuous process, solving the stochastic differential equation

$$
\mathrm{d} X_{t}=\left(\mu_{0}\left(X_{t}\right)+\mathbf{1}(\theta \leq t)\left(\mu_{1}\left(X_{t}\right)-\mu_{0}\left(X_{t}\right)\right)\right) \mathrm{d} t+\sigma\left(X_{t}\right) \mathrm{d} B_{t}
$$

with $X_{0}=x$, where $\mu_{i}(x), i=0,1$, and $\sigma(x)>0$ are some continuously differentiable functions on $(0, \infty)$, satisfying the conditions

$$
\left|\mu_{i}(x)\right|+|\sigma(x)| \leq K(1+|x|) \quad \text { and } \quad 0<\left|\frac{\mu_{1}(x)-\mu_{0}(x)}{\sigma(x)}\right| \leq K
$$

for all $x>0$ and some $K>0$ fixed. In order to facilitate the considerations of the examples below, we assume that the state space of the process $X$ is the positive half-line $(0, \infty)$. It thus follows from [15, Theorem 4.6] that (2.1) admits a unique strong solution under $\theta=s$, and, hence, $\mathbb{P}_{\pi}(X \in \cdot \mid \theta=s)=\mathbb{P}^{s}(X \in \cdot)$ is the distribution law of a time-homogeneous diffusion process started at some $x>0$, with diffusion coefficient $\sigma(x)$ and the drift rate changing from $\mu_{0}(x)$ to $\mu_{1}(x)$ at time $s \in[0, \infty]$. In this case, we may conclude that the probability measure $\mathbb{P}_{\pi}$ has the structure

$$
\mathbb{P}_{\pi}(X \in \cdot)=\pi \mathbb{P}^{0}(X \in \cdot)+(1-\pi) \int_{0}^{\infty} \mathbb{P}^{s}(X \in \cdot) \lambda \mathrm{e}^{-\lambda s} \mathrm{~d} s
$$

for any fixed $\pi \in[0,1)$.

Based upon the continuous observation of the process $X$, our task is to find among the stopping times $\tau$ of $X$ (i.e. stopping times with respect to the natural filtration $\mathcal{F}_{t}=\sigma\left(X_{s} \mid 0 \leq\right.$ $s \leq t$ ) of the process $X$ ) an optimal time at which an alarm should be sounded as closely as possible to the unobservable time of disorder $\theta$. More precisely, the Bayesian quickest detection problem consists of computing the Bayesian risk function

$$
V(\pi)=\inf _{\tau}\left(\mathbb{P}_{\pi}(\tau<\theta)+\mathbb{E}_{\pi}[F(\tau-\theta) \mathbf{1}(\tau \geq \theta)]\right)
$$

and finding the optimal stopping time, called the $\pi$-Bayesian time, at which the infimum is attained in (2.3). Here $\mathbb{P}_{\pi}(\tau<\theta)$ is the probability of a false alarm, and $\mathbb{E}_{\pi}[F(\tau-\theta) \mathbf{1}(\tau \geq \theta)]$ is the expected costs of delay in detecting the disorder correctly (i.e. when $\tau \geq \theta$ ), where the delay penalty function $F(t)$ satisfies the conditions $F(t) \geq 0$ for $t \geq 0$ and $F(t)=0$ for $t \leq 0$. We will further assume that either $F(t)=c t$ or $F(t)=c\left(\mathrm{e}^{\alpha t}-1\right)$ holds in (2.3) for all $t \geq 0$.

Remark 2.1. It was shown in [6], [25], and [30] that, when the Laplace transforms of delay penalty functions are of rational structure, the rewards of the Bayesian quickest detection problems such as in (2.3) can be expressed through finite-dimensional processes called Markovian sufficient statistics. Such (time-homogeneous strong) Markov processes contain all the necessary information to determine the optimal stopping times (see [28, Chapter II, Section 15] for an extensive discussion of this notion). For instance, the function $F(t)=c t^{\delta}$ for $t \geq 0$, with some $c, \delta>0, \delta \in \mathbb{N}$, is of such type, while the assumption $\delta \notin \mathbb{N}$ leads to the appearance of an infinite-dimensional Markovian sufficient statistic in that case. 


\subsection{Likelihood ratio and posterior probability}

In order to derive Markovian sufficient statistics for the problem of (2.3), for the cases of linear and exponential delay penalty functions indicated above, let us define the posterior probability process $\left(\pi_{t}\right)_{t \geq 0}$ by $\pi_{t}=\mathbb{P}\left(\theta \leq t \mid \mathcal{F}_{t}\right)$ for $t \geq 0$. Taking into account the fact that the probability measure $\mathbb{P}^{S}$ is equivalent to $\mathbb{P}_{\pi}$ on $\mathcal{F}_{t}$ by construction for any $s \in[0, \infty]$, using Bayes' formula (see, e.g. [15, Theorem 7.23]), we find that $\left(\pi_{t}\right)_{t \geq 0}$ admits the representation

$$
\pi_{t}=\pi \frac{\mathrm{d}\left(\mathbb{P}^{0} \mid \mathcal{F}_{t}\right)}{\mathrm{d}\left(\mathbb{P}_{\pi} \mid \mathcal{F}_{t}\right)}+(1-\pi) \int_{0}^{t} \frac{\mathrm{d}\left(\mathbb{P}^{s} \mid \mathscr{F}_{t}\right)}{\mathrm{d}\left(\mathbb{P}_{\pi} \mid \mathcal{F}_{t}\right)} \lambda \mathrm{e}^{-\lambda s} \mathrm{~d} s
$$

Moreover, since the measure $\mathbb{P}^{u}$ coincides with $\mathbb{P}^{t}$ on $\mathcal{F}_{t}$ for all $0 \leq t \leq u$, we see that

$$
1-\pi_{t}=(1-\pi) \int_{t}^{\infty} \frac{\mathrm{d}\left(\mathbb{P}^{u} \mid \mathcal{F}_{t}\right)}{\mathrm{d}\left(\mathbb{P}_{\pi} \mid \mathscr{F}_{t}\right)} \lambda \mathrm{e}^{-\lambda u} \mathrm{~d} u=(1-\pi) \mathrm{e}^{-\lambda t} \frac{\mathrm{d}\left(\mathbb{P}^{t} \mid \mathcal{F}_{t}\right)}{\mathrm{d}\left(\mathbb{P}_{\pi} \mid \mathcal{F}_{t}\right)}
$$

is satisfied. By means of Girsanov's theorem for diffusion processes (see, e.g. [15, Theorem 7.19]), it follows from the structure of the observation process $X$ in (2.1) that the likelihood ratio process $L=\left(L_{t}\right)_{t \geq 0}$ defined by

$$
L_{t}=\frac{\mathrm{d}\left(\mathbb{P}^{0} \mid \mathcal{F}_{t}\right)}{\mathrm{d}\left(\mathbb{P}^{t} \mid \mathcal{F}_{t}\right)} \equiv \frac{\mathrm{d}\left(\mathbb{P}^{0} \mid \mathcal{F}_{t}\right)}{\mathrm{d}\left(\mathbb{P}^{\infty} \mid \mathcal{F}_{t}\right)}
$$

admits the representation

$$
L_{t}=\exp \left(\int_{0}^{t} \frac{\mu_{1}\left(X_{s}\right)-\mu_{0}\left(X_{s}\right)}{\sigma^{2}\left(X_{s}\right)} \mathrm{d} X_{s}-\frac{1}{2} \int_{0}^{t} \frac{\mu_{1}^{2}\left(X_{s}\right)-\mu_{0}^{2}\left(X_{s}\right)}{\sigma^{2}\left(X_{s}\right)} \mathrm{d} s\right) .
$$

Hence, it follows from the expressions in (2.6) and (2.7) that the properties

$$
\frac{\mathrm{d}\left(\mathbb{P}^{s} \mid \mathcal{F}_{t}\right)}{\mathrm{d}\left(\mathbb{P}_{\pi} \mid \mathcal{F}_{t}\right)} \frac{\mathrm{d}\left(\mathbb{P}_{\pi} \mid \mathcal{F}_{t}\right)}{\mathrm{d}\left(\mathbb{P}^{t} \mid \mathcal{F}_{t}\right)}=\frac{\mathrm{d}\left(\mathbb{P}^{s} \mid \mathcal{F}_{t}\right)}{\mathrm{d}\left(\mathbb{P}^{0} \mid \mathcal{F}_{t}\right)} \frac{\mathrm{d}\left(\mathbb{P}^{0} \mid \mathcal{F}_{t}\right)}{\mathrm{d}\left(\mathbb{P}^{t} \mid \mathcal{F}_{t}\right)}=\frac{\mathrm{d}\left(\mathbb{P}^{s} \mid \mathcal{F}_{s}\right)}{\mathrm{d}\left(\mathbb{P}^{0} \mid \mathcal{F}_{s}\right)} \frac{\mathrm{d}\left(\mathbb{P}^{0} \mid \mathcal{F}_{t}\right)}{\mathrm{d}\left(\mathbb{P}^{t} \mid \mathcal{F}_{t}\right)} \equiv \frac{L_{t}}{L_{s}}
$$

hold for each $0 \leq s \leq t$. It therefore follows from the representations in (2.4) and (2.5) that the weighted likelihood ratio process $\left(\varphi_{t}\right)_{t \geq 0}$ defined by $\varphi_{t}=\pi_{t} /\left(1-\pi_{t}\right)$ has the form

$$
\varphi_{t}=\mathrm{e}^{\lambda t} L_{t}\left(\frac{\pi}{1-\pi}+\int_{0}^{t} \frac{\lambda \mathrm{e}^{-\lambda s}}{L_{s}} \mathrm{~d} s\right) .
$$

\subsection{Stochastic differential equations}

Applying Itô's formula (see, e.g. [15, Chapter IV, Theorem 4.4] or [22, Chapter IV, Theorem 3.3]) to the expression in (2.7), we find that the process $L$ admits the representation

$$
\mathrm{d} L_{t}=\frac{\mu_{1}\left(X_{t}\right)-\mu_{0}\left(X_{t}\right)}{\sigma^{2}\left(X_{t}\right)} L_{t}\left(\mathrm{~d} X_{t}-\mu_{0}\left(X_{t}\right) \mathrm{d} t\right)
$$

with $L_{0}=1$. Then, using the integration-by-parts formula, we see that the process $\left(\varphi_{t}\right)_{t \geq 0}$ from (2.8) solves the stochastic differential equation

$$
\mathrm{d} \varphi_{t}=\left(\lambda\left(1+\varphi_{t}\right)+\left(\frac{\mu_{1}\left(X_{t}\right)-\mu_{0}\left(X_{t}\right)}{\sigma\left(X_{t}\right)}\right)^{2} \frac{\varphi_{t}^{2}}{1+\varphi_{t}}\right) \mathrm{d} t+\frac{\mu_{1}\left(X_{t}\right)-\mu_{0}\left(X_{t}\right)}{\sigma\left(X_{t}\right)} \varphi_{t} \mathrm{~d} \bar{B}_{t}
$$


with $\varphi_{0}=\varphi \equiv \pi /(1-\pi)$. Hence, using Itô's formula again, we find that the process $\left(\pi_{t}\right)_{t \geq 0}$ admits the representation

$$
\mathrm{d} \pi_{t}=\lambda\left(1-\pi_{t}\right) \mathrm{d} t+\frac{\mu_{1}\left(X_{t}\right)-\mu_{0}\left(X_{t}\right)}{\sigma\left(X_{t}\right)} \pi_{t}\left(1-\pi_{t}\right) \mathrm{d} \bar{B}_{t}
$$

with $\pi_{0}=\pi$. Here, the innovation process $\bar{B}=\left(\bar{B}_{t}\right)_{t \geq 0}$ defined by

$$
\bar{B}_{t}=\int_{0}^{t} \frac{\mathrm{d} X_{s}}{\sigma\left(X_{s}\right)}-\int_{0}^{t}\left(\frac{\mu_{0}\left(X_{s}\right)}{\sigma\left(X_{s}\right)}+\pi_{s} \frac{\mu_{1}\left(X_{s}\right)-\mu_{0}\left(X_{s}\right)}{\sigma\left(X_{s}\right)}\right) \mathrm{d} s
$$

is a standard Brownian motion under the measure $\mathbb{P}_{\pi}$, with respect to the filtration $\left(\mathcal{F}_{t}\right)_{t \geq 0}$, according to P. Lévy's characterization theorem (see, e.g. [15, Theorem 4.1] or [22, Chapter IV, Theorem 3.6]). It thus follows from (2.11) that the process $X$ admits the representation

$$
\mathrm{d} X_{t}=\left(\mu_{0}\left(X_{t}\right)+\pi_{t}\left(\mu_{1}\left(X_{t}\right)-\mu_{0}\left(X_{t}\right)\right)\right) \mathrm{d} t+\sigma\left(X_{t}\right) \mathrm{d} \bar{B}_{t}
$$

with $X_{0}=x$. Taking into account the assumptions in (2.2), we may conclude by virtue of the remark to [15, Chapter IV, Theorem 4.6] (see also [16, Chapter V, Theorem 5.2.1]) that the processes $\left(\pi_{t}, X_{t}\right)_{t \geq 0}$ and $\left(\varphi_{t}, X_{t}\right)_{t \geq 0}$ turn out to be unique strong solutions of the corresponding systems of stochastic differential equations in (2.9), (2.10), and (2.12). According to [16, Chapter VII, Theorem 7.2.4], such processes have the (time-homogeneous strong) Markov property with respect to their natural filtration, which inherently coincides with $\left(\mathcal{F}_{t}\right)_{t \geq 0}$.

\subsection{Some examples}

Let us now present some expressions for the Bayesian risk functions and the appropriate Markovian sufficient statistics in the corresponding quickest disorder detection problems for observable diffusion processes.

Example 2.1. Assume that we have $F(t)=c t$ with some $c>0$ fixed (see [26], [27], [28, Chapter IV], and [18, Chapter VI, Section 22]). It is then shown by means of standard arguments from [28, Chapter IV, Section 3] that the Bayesian risk function $V(\pi)$ in (2.3) admits the representation

$$
V^{\prime}(\pi, \varphi, x)=\inf _{\tau} \mathbb{E}_{\pi, \varphi, x}\left[1-\pi_{\tau}+\int_{0}^{\tau}\left(1-\pi_{t}\right) c \varphi_{t} \mathrm{~d} t\right],
$$

where the infimum is taken over all stopping times $\tau$ such that $\mathbb{E}_{\pi, \varphi, x} \tau<\infty$ holds. Here $\mathbb{P}_{\pi, \varphi, x}$ is a measure of the diffusion process $\left(\pi_{t}, \varphi_{t}, X_{t}\right)_{t \geq 0}$, started at some $(\pi, \varphi, x) \in[0,1) \times$ $[0, \infty) \times(0, \infty)$ and solving (2.9), (2.10), and (2.12), which is a Markovian sufficient statistic in the problem. Note that, according to the arguments in [7], the value function in (2.13) can be studied as one of the appropriate optimal stopping problems for the equivalent Markovian sufficient statistics $\left(\pi_{t}, X_{t}\right)_{t \geq 0}$ or $\left(\varphi_{t}, X_{t}\right)_{t \geq 0}$, under an auxiliary probability measure.

Example 2.2. Assume now that $F(t)=c\left(\mathrm{e}^{\alpha t}-1\right)$ with some fixed $c, \alpha>0$ (see [25, Example 4], [19], [4], and [1]). It can be shown following the schema of arguments from [1] that the Bayesian risk function $V(\pi)$ in $(2.3)$ admits the representation

$$
V_{*}(\pi, \phi, x)=\inf _{\tau} \mathbb{E}_{\pi, \phi, x}\left[1-\pi_{\tau}+\int_{0}^{\tau}\left(1-\pi_{t}\right) c \alpha \phi_{t} \mathrm{~d} t\right],
$$


where the infimum is taken over all stopping times $\tau$ such that the integral above has a finite expectation, so that $\mathbb{E}_{\pi, \phi, x} \tau<\infty$ holds. Here, the weighted likelihood ratio process $\left(\phi_{t}\right)_{t \geq 0}$ defined by

$$
\phi_{t}=\mathrm{e}^{(\alpha+\lambda) t} L_{t}\left(\frac{\pi}{1-\pi}+\int_{0}^{t} \frac{\lambda \mathrm{e}^{-(\alpha+\lambda) s}}{L_{s}} \mathrm{~d} s\right)
$$

solves the stochastic differential equation

$$
\mathrm{d} \phi_{t}=\left(\lambda+(\lambda+\alpha) \phi_{t}+\left(\frac{\mu_{1}\left(X_{t}\right)-\mu_{0}\left(X_{t}\right)}{\sigma\left(X_{t}\right)}\right)^{2} \pi_{t} \phi_{t}\right) \mathrm{d} t+\frac{\mu_{1}\left(X_{t}\right)-\mu_{0}\left(X_{t}\right)}{\sigma\left(X_{t}\right)} \phi_{t} \mathrm{~d} \bar{B}_{t}
$$

with $\phi_{0}=\phi \equiv \pi /(1-\pi)$. In this case, $\mathbb{P}_{\pi, \phi, x}$ is a measure of the diffusion process $\left(\pi_{t}, \phi_{t}, X_{t}\right)_{t \geq 0}$, started at some $(\pi, \phi, x) \in[0,1) \times[0, \infty) \times(0, \infty)$ and solving $(2.10),(2.12)$, and (2.16), which is a Markovian sufficient statistic in the problem. Note that, according to the arguments in [1] and [2], the value function in (2.14) can be studied as one of the appropriate optimal stopping problems for the Markovian sufficient statistic $\left(\phi_{t}, X_{t}\right)_{t \geq 0}$, under an auxiliary probability measure.

\section{The case of exponential delay penalty costs}

In this section we formulate and prove the main assertions of the paper, which are related to the quickest detection problem with exponential delay penalty costs of Example 2.2.

\subsection{The structure of the optimal stopping time}

By means of the results of general theory of optimal stopping (see, e.g. [28, Chapter III] or [18, Chapter I, Section 2.1]), it follows from the structure of the reward functional in (2.14) that the optimal stopping time is given by

$$
\tau_{*}=\inf \left\{t \geq 0 \mid V_{*}\left(\pi_{t}, \phi_{t}, X_{t}\right)=1-\pi_{t}\right\}
$$

whenever the corresponding integral there is of finite expectation, so that $\mathbb{E}_{\pi, \phi, x} \tau_{*}<\infty$ holds. In order to specify the structure of the stopping time in (3.1), we follow the arguments from [10, Subsection 2.5] and use Itô's formula to obtain

$$
1-\pi_{t}=1-\pi-\int_{0}^{t} \lambda\left(1-\pi_{s}\right) \mathrm{d} s+N_{t}
$$

where the process $N=\left(N_{t}\right)_{t \geq 0}$ defined by

$$
N_{t}=-\int_{0}^{t} \frac{\mu_{1}\left(X_{s}\right)-\mu_{0}\left(X_{s}\right)}{\sigma\left(X_{s}\right)} \pi_{s}\left(1-\pi_{s}\right) \mathrm{d} \bar{B}_{s}
$$

is a continuous local martingale under $\mathbb{P}_{\pi, \phi, x}$. It follows directly from (3.2) that the process $\left(N_{\tau \wedge t}\right)_{t \geq 0}$ is a uniformly integrable martingale for any stopping time $\tau$ satisfying $\mathbb{E}_{\pi, \phi, x} \tau<$ $\infty$. Then, applying Doob's optional sampling theorem (see, e.g. [15, Theorem 3.6] or [22, Chapter II, Theorem 3.2]), we find from the expression in (3.2) that

$$
\mathbb{E}_{\pi, \phi, x}\left[1-\pi_{\tau}+\int_{0}^{\tau}\left(1-\pi_{t}\right) c \alpha \phi_{t} \mathrm{~d} t\right]=1-\pi+\mathbb{E}_{\pi, \phi, x} \int_{0}^{\tau}\left(1-\pi_{t}\right)\left(c \alpha \phi_{t}-\lambda\right) \mathrm{d} t
$$

holds for all $(\pi, \phi, x) \in[0,1) \times[0, \infty) \times(0, \infty)$ and any $\tau$ such that $\mathbb{E}_{\pi, \phi, x} \tau<\infty$. Taking into account the structure of the reward in (2.14), it is seen from (3.3) that it is never optimal 
to stop when $\phi_{t}<\lambda /(c \alpha)$ for any $t \geq 0$. This shows that all the points $(\pi, \phi, x)$ such that $\phi<\lambda /(c \alpha)$ belong to the continuation region

$$
C_{*}=\left\{(\pi, \phi, x) \in[0,1) \times[0, \infty) \times(0, \infty) \mid V_{*}(\pi, \phi, x)<1-\pi\right\} .
$$

\subsection{The structure of the continuation region}

In order to describe the structure of the set in (3.4), let us fix some $(\pi, \phi, x) \in C_{*}$ and denote by $\tau_{*}=\tau_{*}(\pi, \phi, x)$ the optimal stopping time in problem (2.14). Then, by means of the general optimal stopping theory for Markov processes (see, e.g. [28, Chapter III] or [18, Chapter I, Section 2.2]), we conclude that

$$
V_{*}(\pi, \phi, x)=\mathbb{E}_{\pi, \phi, x}\left[1-\pi_{\tau_{*}}+\int_{0}^{\tau_{*}}\left(1-\pi_{t}\right) c \alpha \phi_{t} \mathrm{~d} t\right]<1-\pi
$$

holds. Hence, taking any $\phi^{\prime}$ such that $\phi^{\prime}<\phi$ and using the explicit expression for the process $\left(\phi_{t}\right)_{t \geq 0}$ through its starting point $\phi \equiv \pi /(1-\pi)$ in (2.15), we find from (2.14) that the inequalities

$$
\begin{aligned}
V_{*}\left(\pi, \phi^{\prime}, x\right) & \leq \mathbb{E}_{\pi, \phi^{\prime}, x}\left[1-\pi_{\tau_{*}}+\int_{0}^{\tau_{*}}\left(1-\pi_{t}\right) c \alpha \phi_{t} \mathrm{~d} t\right] \\
& \leq \mathbb{E}_{\pi, \phi, x}\left[1-\pi_{\tau_{*}}+\int_{0}^{\tau_{*}}\left(1-\pi_{t}\right) c \alpha \phi_{t} \mathrm{~d} t\right]
\end{aligned}
$$

are satisfied. Thus, by virtue of the inequality in (3.5), we see that $\left(\pi, \phi^{\prime}, x\right) \in C_{*}$. Taking into account the multiplicative structure of the integrand in (2.14), we can therefore extend the approach used in [19], [4], and [1], and further assume that there exists a function $g_{*}(x)$ such that $0<\lambda /(c \alpha) \leq g_{*}(x)$ for $x>0$, and the continuation region in (3.4) for the optimal stopping problem of (2.14) takes the form

$$
C_{*}=\left\{(\pi, \phi, x) \in[0,1) \times[0, \infty) \times(0, \infty) \mid \phi<g_{*}(x)\right\},
$$

so that the corresponding stopping region is the closure of the set

$$
D_{*}=\left\{(\pi, \phi, x) \in[0,1) \times[0, \infty) \times(0, \infty) \mid \phi>g_{*}(x)\right\} .
$$

\subsection{The optimal stopping boundary}

In order to characterize the behavior of the boundary $g_{*}(x)$ in (3.6)-(3.7), we apply the integration-by-parts formula to the processes from (2.16) and (3.2) to conclude that the expression

$$
\begin{aligned}
\int_{0}^{\tau_{*}}\left(1-\pi_{t}\right)\left(c \alpha \phi_{t}-\lambda\right) \mathrm{d} t \\
\quad=\int_{0}^{\tau_{*}}\left((1-\pi)\left(\frac{c \alpha \pi}{1-\pi}-\lambda\right)+\int_{0}^{t}\left(c \alpha\left(\lambda+\alpha \phi_{s}\right)+\lambda^{2}\right)\left(1-\pi_{s}\right) \mathrm{d} s+N_{t}^{*}\right) \mathrm{d} t
\end{aligned}
$$

holds for the optimal stopping time $\tau_{*}=\tau_{*}(\pi, \phi, x)$ in (2.14) such that $(\pi, \phi, x) \in C_{*}$. Here, the process $N^{*}=\left(N_{t}^{*}\right)_{t \geq 0}$ defined by

$$
N_{t}^{*}=\int_{0}^{t} \frac{\mu_{1}\left(X_{s}\right)-\mu_{0}\left(X_{s}\right)}{\sigma\left(X_{s}\right)}\left(c \alpha\left(1-\pi_{s}\right) \phi_{s}+\lambda \pi_{s}\right) \mathrm{d} \bar{B}_{s}
$$


is a continuous local martingale under $\mathbb{P}_{\pi, \phi, x}$, and its quadratic variation process is given by

$$
\left\langle N^{*}\right\rangle_{t}=\int_{0}^{t}\left(\frac{\mu_{1}\left(X_{S}\right)-\mu_{0}\left(X_{s}\right)}{\sigma\left(X_{s}\right)}\right)^{2}\left(c \alpha\left(1-\pi_{s}\right) \phi_{s}+\lambda \pi_{s}\right)^{2} \mathrm{~d} s .
$$

Let us now take $x^{\prime}>0$ such that $x<x^{\prime}$, and define the process $X^{\prime}=\left(X_{t}^{\prime}\right)_{t \geq 0}$ as a solution of (2.12) started at $x^{\prime}$. It follows from the comparison results in [32] for solutions of multidimensional stochastic differential equations that the property $X_{t} \leq X_{t}^{\prime}$ (P्P-almost surely) holds, so that the process $N^{*}$ has a larger quadratic variation when the process $X$ starts at $x^{\prime}$ rather than at $x$, whenever the so-called signal-to-noise ratio function $\rho(x)$ given by

$$
\rho(x)=\left(\frac{\mu_{1}(x)-\mu_{0}(x)}{\sigma(x)}\right)^{2}
$$

is increasing on $(0, \infty)$. Suppose that the process $\left(N_{\tau_{*} \wedge t}^{*}\right)_{t \geq 0}$ is a uniformly integrable martingale under both $\mathbb{P}_{\pi, \phi, x}$ and $\mathbb{P}_{\pi, \phi, x^{\prime}}$, which is the case for the process $\left(M_{\tau_{*} \wedge t}\right)_{t \geq 0}$ from (3.37) below under the conditions of Lemma 3.2 below. Then, it follows from the assumption that the integral in (2.14) taken up to the optimal stopping time $\tau_{*}$ is of finite expectation and the third inequality in (2.2) that the integrals on the both sides of the expression in (3.8) are of finite expectation too. Hence, taking into account the fact that $\tau_{*}$ has the structure of the first time the process $\left(\phi_{t}\right)_{t \geq 0}$ hits an upper boundary, by virtue of arguments similar to those used in the case of constant coefficients $\mu_{i}, i=0,1$, and $\sigma>0$ in (2.1), we conclude that the inequality

$$
\begin{aligned}
\mathbb{E}_{\pi, \phi, x^{\prime}} & \int_{0}^{\tau_{*}}\left((1-\pi)\left(\frac{c \alpha \pi}{1-\pi}-\lambda\right)+\int_{0}^{t}\left(c \alpha\left(\lambda+\alpha \phi_{s}\right)+\lambda^{2}\right)\left(1-\pi_{s}\right) \mathrm{d} s+N_{t}^{*}\right) \mathrm{d} t \\
\leq & \mathbb{E}_{\pi, \phi, x} \int_{0}^{\tau_{*}}\left((1-\pi)\left(\frac{c \alpha \pi}{1-\pi}-\lambda\right)+\int_{0}^{t}\left(c \alpha\left(\lambda+\alpha \phi_{s}\right)+\lambda^{2}\right)\left(1-\pi_{s}\right) \mathrm{d} s+N_{t}^{*}\right) \mathrm{d} t
\end{aligned}
$$

is satisfied, whenever $\rho(x)$ is increasing on $(0, \infty)$. Finally, gathering expressions (3.3), (3.8), and (3.11), we see that

$$
\begin{aligned}
V_{*}\left(\pi, \phi, x^{\prime}\right)-(1-\pi) & \leq \mathbb{E}_{\pi, \phi, x^{\prime}} \int_{0}^{\tau_{*}}\left(1-\pi_{t}\right)\left(c \alpha \phi_{t}-\lambda\right) \mathrm{d} t \\
& \leq \mathbb{E}_{\pi, \phi, x} \int_{0}^{\tau_{*}}\left(1-\pi_{t}\right)\left(c \alpha \phi_{t}-\lambda\right) \mathrm{d} t \\
& =V_{*}(\pi, \phi, x)-(1-\pi)
\end{aligned}
$$

holds, whenever $\rho(x)$ is increasing on $(0, \infty)$. By virtue of the inequalities in (3.12), we may therefore conclude that $\left(\pi, \phi, x^{\prime}\right) \in C_{*}$, so that the boundary $g_{*}(x)$ is increasing or decreasing in (3.6)-(3.7), whenever $\rho(x)$ is increasing or decreasing on $(0, \infty)$, respectively.

Summarizing the facts proved above, we are now ready to formulate the following assertion.

Lemma 3.1. Suppose that $\mu_{i}(x), i=0,1$, and $\sigma(x)>0$ are continuously differentiable functions on $(0, \infty)$ in (2.1) satisfying (2.2). Assume that the process $\left(N_{\tau_{*} \wedge t}^{*}\right)_{t \geq 0}$ from (3.9) is a uniformly integrable martingale. Then the optimal Bayesian time of alarm $\tau_{*}$ in the quickest disorder detection problem of (2.14) has the structure

$$
\tau_{*}=\inf \left\{t \geq 0 \mid \phi_{t} \geq g_{*}\left(X_{t}\right)\right\}
$$


whenever the corresponding integral is of finite expectation, so that $\mathbb{E}_{\pi, \phi, x} \tau_{*}<\infty$ holds for all $(\pi, \phi, x) \in[0,1) \times[0, \infty) \times(0, \infty)$, and $\tau_{*}=0$ otherwise. Moreover, the property

$$
g_{*}(x):(0, \infty) \rightarrow\left(\frac{\lambda}{c \alpha}, \infty\right) \text { is increasing/decreasing if } \rho(x) \text { is increasing/decreasing }
$$

holds with $\rho(x)$ defined in (3.10) for all $x>0$.

\subsection{The free-boundary problem}

By means of standard arguments based on the application of Itô's formula, we show that the infinitesimal operator $\mathbb{L}_{(\pi, \phi, X)}$ of the process $\left(\pi_{t}, \phi_{t}, X_{t}\right)_{t \geq 0}$ from (2.16), (2.10), and (2.12) has the structure

$$
\begin{aligned}
\mathbb{L}_{(\pi, \phi, X)}= & \lambda(1-\pi) \frac{\partial}{\partial \pi}+\left(\lambda+(\lambda+\alpha) \phi+\left(\frac{\mu_{1}(x)-\mu_{0}(x)}{\sigma(x)}\right)^{2} \pi \phi\right) \frac{\partial}{\partial \phi} \\
& +\left(\mu_{0}(x)+\left(\mu_{1}(x)-\mu_{0}(x)\right) \pi\right) \frac{\partial}{\partial x} \\
& +\left(\mu_{1}(x)-\mu_{0}(x)\right)\left(\pi(1-\pi) \frac{\partial^{2}}{\partial \pi \partial x}+\phi \frac{\partial^{2}}{\partial \phi \partial x}\right) \\
& +\frac{1}{2}\left(\frac{\mu_{1}(x)-\mu_{0}(x)}{\sigma(x)}\right)^{2}\left(\pi^{2}(1-\pi)^{2} \frac{\partial^{2}}{\partial \pi^{2}}+2 \pi(1-\pi) \phi \frac{\partial^{2}}{\partial \pi \partial \phi}+\phi^{2} \frac{\partial^{2}}{\partial \phi^{2}}\right) \\
& +\frac{1}{2} \sigma^{2}(x) \frac{\partial^{2}}{\partial x^{2}}
\end{aligned}
$$

for all $(\pi, \phi, x) \in[0,1) \times[0, \infty) \times(0, \infty)$.

According to the results of the general theory of optimal stopping problems for continuoustime Markov processes (see, e.g. [12], [18, Chapter IV, Section 8], and [28, Chapter III, Section 8]), we can formulate the associated free-boundary problem for the unknown value function $V_{*}(\pi, \phi, x)$ from (2.14) and the boundary $g_{*}(x)$ from (3.13):

$$
\begin{gathered}
\left(\mathbb{L}_{(\pi, \phi, X)} V\right)(\pi, \phi, x)=-(1-\pi) c \alpha \phi \quad \text { for }(\pi, \phi, x) \in C, \\
\left.V(\pi, \phi, x)\right|_{\phi=g(x)-=1-\pi} \quad \text { (instantaneous stopping), } \\
V(\pi, \phi, x)=1-\pi \quad \text { for }(\pi, \phi, x) \in D, \\
V(\pi, \phi, x)<1-\pi \quad \text { for }(\pi, \phi, x) \in C, \\
\left(\mathbb{L}_{(\pi, \phi, X)} V\right)(\pi, \phi, x)>-(1-\pi) c \alpha \phi \quad \text { for }(\pi, \phi, x) \in D .
\end{gathered}
$$

Here $C$ and $D$ are defined as $C_{*}$ and $D_{*}$ in (3.6) and (3.7) with $g(x)$ instead of $g_{*}(x)$, and the condition in (3.17) is satisfied for all $\pi \in[0,1)$ and $x>0$.

Note that the superharmonic characterization of the value function (see [8], [18, Chapter IV, Section 9] and [28, Chapter III, Section 8]) implies that $V_{*}(\pi, \phi, x)$ from (2.14) is the largest function satisfying (3.16)-(3.20) with the boundary $g_{*}(x)$.

Remark 3.1. Observe that, since the system in (3.16)-(3.20) admits multiple solutions, we need to find some additional conditions which would specify the appropriate solution providing the value function and the optimal stopping boundary for the initial problem of (2.14). In order to 
derive such conditions, we will reduce the operator in (3.15) to the normal form. We also note that the fact that the stochastic differential equations for the posterior probability, the weighted likelihood ratio, and the observation process in (2.10), (2.16), and (2.12), respectively, are driven by the same (one-dimensional) innovation Brownian motion yields the property that the infinitesimal operator in (3.15) turns out to be of parabolic type.

\subsection{The change of variables}

In order to find the normal form of the operator in (3.15) and formulate the appropriate optimal stopping and free-boundary problem, we use the one-to-one correspondence transformation of processes proposed by Kolmogorov in [13]. For this, let us define the process $Y=\left(Y_{t}\right)_{t \geq 0}$ by

$$
Y_{t}=\log \phi_{t}-\int_{z}^{X_{t}} \frac{\mu_{1}(w)-\mu_{0}(w)}{\sigma^{2}(w)} \mathrm{d} w
$$

for all $t \geq 0$ and any fixed $z>0$. Then, taking into account the assumption that the functions $\mu_{i}(x), i=0,1$, and $\sigma(x)$ are continuously differentiable on $(0, \infty)$, by means of Itô's formula, we conclude that the process $Y$ admits the representation

$$
\mathrm{d} Y_{t}=\left(\frac{\lambda}{\phi_{t}}+\lambda+\alpha-\frac{\sigma^{2}\left(X_{t}\right)}{2}\left[\frac{\mu_{1}^{2}\left(X_{t}\right)-\mu_{0}^{2}\left(X_{t}\right)}{\sigma^{4}\left(X_{t}\right)}+\left.\frac{\partial}{\partial x}\left(\frac{\mu_{1}(x)-\mu_{0}(x)}{\sigma^{2}(x)}\right)\right|_{x=X_{t}}\right]\right) \mathrm{d} t
$$

with $Y_{0}=y$ and

$$
y=\log \phi-\int_{z}^{x} \frac{\mu_{1}(w)-\mu_{0}(w)}{\sigma^{2}(w)} \mathrm{d} w
$$

for any fixed $z>0$. It is seen from (3.22) that the process $Y$ started at $y \in \mathbb{R}$ is of bounded variation. By virtue of the second inequality in (2.2), it follows from the relation in (3.21) that there exists a one-to-one correspondence between the processes $(\pi, \phi, X)$ and $(\pi, \phi, Y)$. Hence, for any fixed $z>0$, the value function $V_{*}(\pi, \phi, x)$ from (2.14) is equal to the following optimal stopping problem:

$$
U_{*}(\pi, \phi, y)=\inf _{\tau} \mathbb{E}_{\pi, \phi, y}\left[1-\pi_{\tau}+\int_{0}^{\tau}\left(1-\pi_{t}\right) c \alpha \phi_{t} \mathrm{~d} t\right]
$$

with the infimum taken over all stopping times $\tau$ such that the integral is of finite expectation, so that $\mathbb{E}_{\pi, \phi, y} \tau<\infty$ holds. Here $\mathbb{P}_{\pi, \phi, y}$ is a measure of the diffusion process $\left(\pi_{t}, \phi_{t}, Y_{t}\right)_{t \geq 0}$ started at some $(\pi, \phi, y) \in[0,1) \times(0, \infty) \times \mathbb{R}$ from (2.8), (2.15), and (3.21). It thus follows from (3.6)-(3.7) that there exists a continuous function $h_{*}(y)$ such that $0<\lambda /(c \alpha) \leq h_{*}(y)$ holds for $y \in \mathbb{R}$, and the optimal stopping time in problem (3.24) has the structure

$$
\tau_{*}=\inf \left\{t \geq 0 \mid \phi_{t} \geq h_{*}\left(Y_{t}\right)\right\}
$$

whenever the corresponding integral is of finite expectation, so that $\mathbb{E}_{\pi, \phi, y} \tau_{*}<\infty$ holds, and $\tau_{*}=0$ otherwise. 


\subsection{The free-boundary problem}

Standard arguments then show that the infinitesimal operator $\mathbb{L}_{(\pi, \phi, Y)}$ of the process $(\pi, \phi, Y)$ solving (2.10), (2.16), and (3.22) has the structure

$$
\begin{aligned}
\mathbb{L}_{(\pi, \phi, Y)}= & \lambda(1-\pi) \frac{\partial}{\partial \pi}+\left(\lambda+(\lambda+\alpha) \phi+\left(\frac{\mu_{1}(x(\phi, y))-\mu_{0}(x(\phi, y))}{\sigma(x(\phi, y))}\right)^{2} \pi \phi\right) \frac{\partial}{\partial \phi} \\
+ & \frac{1}{2}\left(\frac{\mu_{1}(x(\phi, y))-\mu_{0}(x(\phi, y))}{\sigma(x(\phi, y))}\right)^{2} \\
& \times\left(\pi^{2}(1-\pi)^{2} \frac{\partial^{2}}{\partial \pi^{2}}+2 \pi(1-\pi) \phi \frac{\partial^{2}}{\partial \pi \partial \phi}+\phi^{2} \frac{\partial^{2}}{\partial \phi^{2}}\right) \\
+ & \left(\frac{\lambda}{\phi}+\lambda+\alpha-\frac{\sigma^{2}(x(\phi, y))}{2}\left[\frac{\mu_{1}^{2}(x(\phi, y))-\mu_{0}^{2}(x(\phi, y))}{\sigma^{4}(x(\phi, y))}\right.\right. \\
& \left.\left.+\left.\frac{\partial}{\partial x}\left(\frac{\mu_{1}(x)-\mu_{0}(x)}{\sigma^{2}(x)}\right)\right|_{x=x(\phi, y)}\right]\right) \frac{\partial}{\partial y}
\end{aligned}
$$

for all $(\pi, \phi, y) \in[0,1) \times(0, \infty) \times \mathbb{R}$. Here, because of the second inequality in (2.2), the expression for $x(\phi, y) \equiv x(\phi, y ; z)$ is uniquely determined by the relation in (3.23) for any $z>0$.

We are now ready to formulate the associated free-boundary problem for the unknown value function $U_{*}(\pi, \phi, y) \equiv U_{*}(\pi, \phi, y ; z)$ from (3.24) and the boundary $h_{*}(y) \equiv h_{*}(y ; z)$ from (3.25):

$$
\begin{gathered}
\left(\mathbb{L}_{(\pi, \phi, Y)} U\right)(\pi, \phi, y)=-(1-\pi) c \alpha \phi \quad \text { for } \phi<h(y), \\
\left.U(\pi, \phi, y)\right|_{\phi=h(y)-}=1-\pi \quad \text { (instantaneous stopping), } \\
U(\pi, \phi, y)=1-\pi \quad \text { for } \phi>h(y), \\
U(\pi, \phi, y)<1-\pi \quad \text { for } \phi<h(y), \\
\left(\mathbb{L}_{(\pi, \phi, Y)} U\right)(\pi, \phi, y)>-(1-\pi) c \alpha \phi \quad \text { for } \phi>h(y) .
\end{gathered}
$$

The condition in (3.28) is satisfied for all $\pi \in[0,1)$ and $y \in \mathbb{R}$. Moreover, we assume that the conditions

$$
\begin{gathered}
\left.\frac{\partial U}{\partial \phi}(\pi, \phi, y)\right|_{\phi=h(y)-}=0 \quad \text { (smooth fit) }, \\
\left.\frac{\partial U}{\partial \phi}(\pi, \phi, y)\right|_{\phi=0+} \quad \text { is finite }
\end{gathered}
$$

hold and that the one-sided derivative

$$
\left.\frac{\partial U}{\partial y}(\pi, \phi, y)\right|_{\phi=h(y)-} \text { exists }
$$

for all $\pi \in(0,1), y \in \mathbb{R}$, and any fixed $z>0$.

We further search for solutions of the parabolic-type free-boundary problem in (3.27)-(3.31) satisfying the conditions in (3.32)-(3.34), and such that the resulting boundaries are continuous and of bounded variation. Since such free-boundary problems cannot, in general, be solved explicitly, the existence and uniqueness of classical as well as viscosity solutions of the related variational inequalities and their connection with the optimal stopping problems have been 
extensively studied in the literature (see, e.g. [9], [5], [14], or [16]). It particularly follows from the results of [9, Chapter XVI, Theorem 11.1] as well as [14, Chapter V, Section 3, Theorem 14] with [14, Chapter VI, Section 4, Theorem 12] that the free-boundary problem of (3.27)-(3.31) with (3.32)-(3.34) admits a unique solution.

\subsection{Verification lemma}

We continue with the following verification assertion related to the free-boundary problem in (3.27)-(3.34).

Lemma 3.2. Suppose that $\mu_{i}(x), i=0,1$, and $\sigma(x)>0$ are continuously differentiable functions on $(0, \infty)$ in (2.1) satisfying (2.2). Assume that the function $U\left(\pi, \phi, y ; h_{*}(y)\right) \equiv$ $(1-\pi) H\left(\phi, y ; h_{*}(y)\right)$ and the continuous boundary of bounded variation $h_{*}(y)$ form a unique solution of the free-boundary problem in (3.27)-(3.31) satisfying conditions (3.32)-(3.34). Then the value function of the optimal stopping problem in (3.24) takes the form

$$
U_{*}(\pi, \phi, y)= \begin{cases}(1-\pi) H\left(\phi, y ; h_{*}(y)\right) & \text { if } 0 \leq \phi<h_{*}(y), \\ 1-\pi & \text { if } \phi \geq h_{*}(y),\end{cases}
$$

and $h_{*}(y)$ provides the optimal stopping boundary for (3.25), whenever the corresponding integral is of finite expectation, so that $\mathbb{E}_{\pi, \phi, y} \tau_{*}<\infty$ holds for all $(\pi, \phi, y) \in[0,1) \times$ $(0, \infty) \times \mathbb{R}$.

Proof. Let us denote by $U(\pi, \phi, y)$ the right-hand side of (3.35). Hence, applying the change-of-variable formula with local time on surfaces from [17] to $U(\pi, \phi, y)$ and $h_{*}(y)$, and taking into account the smooth-fit condition in (3.32), we obtain

$$
U\left(\pi_{t}, \phi_{t}, Y_{t}\right)=U(\pi, \phi, y)+\int_{0}^{t}\left(\mathbb{L}_{(\pi, \phi, Y)} U\right)\left(\pi_{s}, \phi_{s}, Y_{S}\right) \mathbf{1}\left(\phi_{s} \neq h_{*}\left(Y_{s}\right)\right) \mathrm{d} s+M_{t},
$$

where the process $M=\left(M_{t}\right)_{t \geq 0}$ defined by

$$
\begin{aligned}
M_{t}= & \int_{0}^{t} \frac{\partial U}{\partial \pi}\left(\pi_{s}, \phi_{s}, Y_{s}\right) \frac{\mu_{1}\left(X_{s}\right)-\mu_{0}\left(X_{s}\right)}{\sigma\left(X_{s}\right)} \pi_{s}\left(1-\pi_{s}\right) \mathrm{d} \bar{B}_{s} \\
& +\int_{0}^{t} \frac{\partial U}{\partial \phi}\left(\pi_{s}, \phi_{s}, Y_{s}\right) \frac{\mu_{1}\left(X_{s}\right)-\mu_{0}\left(X_{s}\right)}{\sigma\left(X_{S}\right)} \phi_{s} \mathrm{~d} \bar{B}_{s}
\end{aligned}
$$

is a continuous local martingale under $\mathbb{P}_{\pi, \phi, y}$ with respect to $\left(\mathcal{F}_{t}\right)_{t \geq 0}$.

It follows from (3.27) and conditions (3.29)-(3.30) that the inequality in (3.31) and, thus, $\left(\mathbb{L}_{(\pi, \phi, Y)} U\right)(\pi, \phi, y) \geq-(1-\pi) c \alpha \phi$ hold for any $(\pi, \phi, y) \in[0,1) \times(0, \infty) \times \mathbb{R}$ such that $\phi \neq h_{*}(y)$, and that $U(\pi, \phi, y) \leq 1-\pi$ is satisfied for all $(\pi, \phi, y) \in[0,1) \times(0, \infty) \times \mathbb{R}$. Recall the assumption that the boundary $h_{*}(y)$ is continuous and of bounded variation and the fact that the process $Y$ from (3.21) is of bounded variation too. We thus conclude from the assumption of continuous differentiability of the functions $\mu_{i}(x), i=0,1$, and $\sigma(x)$ that the time spent by the process $\left(\phi_{t}\right)_{t \geq 0}$ at the boundary $h_{*}(Y)$ is of Lebesgue measure 0 , so that the indicator which appears in (3.36) can be ignored. Hence, it follows from (3.36) that the inequalities

$$
\begin{aligned}
1-\pi_{\tau}+\int_{0}^{\tau}\left(1-\pi_{t}\right) c \alpha \phi_{t} \mathrm{~d} t & \geq U\left(\pi_{\tau}, \phi_{\tau}, Y_{\tau}\right)+\int_{0}^{\tau}\left(1-\pi_{t}\right) c \alpha \phi_{t} \mathrm{~d} t \\
& \geq U(\pi, \phi, y)+M_{\tau}
\end{aligned}
$$

hold for any stopping time $\tau$ of the process $(\pi, \phi, Y)$ started at $(\pi, \phi, y) \in[0,1) \times(0, \infty) \times \mathbb{R}$. 
Let $\left(\tau_{n}\right)_{n \in \mathbb{N}}$ be an arbitrary localizing sequence of stopping times for the processes $M$. Taking expectations with respect to the probability measure $\mathbb{P}_{\pi, \phi, y}$ in (3.38), by means of Doob's optional sampling theorem, we find that the inequalities

$$
\begin{aligned}
& \mathbb{E}_{\pi, \phi, y} {\left[1-\pi_{\tau \wedge \tau_{n}}+\int_{0}^{\tau \wedge \tau_{n}}\left(1-\pi_{t}\right) c \alpha \phi_{t} \mathrm{~d} t\right] } \\
& \geq \mathbb{E}_{\pi, \phi, y}\left[U\left(\pi_{\tau \wedge \tau_{n}}, \phi_{\tau \wedge \tau_{n}}, Y_{\tau \wedge \tau_{n}}\right)+\int_{0}^{\tau \wedge \tau_{n}}\left(1-\pi_{t}\right) c \alpha \phi_{t} \mathrm{~d} t\right] \\
& \geq U(\pi, \phi, y)+\mathbb{E}_{\pi, \phi, y} M_{\tau \wedge \tau_{n}} \\
&=U(\pi, \phi, y)
\end{aligned}
$$

hold for all $(\pi, \phi, y) \in[0,1) \times(0, \infty) \times \mathbb{R}$. Hence, letting $n$ go to $\infty$ and using Fatou's lemma, we obtain

$$
\begin{aligned}
\mathbb{E}_{\pi, \phi, y}\left[1-\pi_{\tau}+\int_{0}^{\tau}\left(1-\pi_{t}\right) c \alpha \phi_{t} \mathrm{~d} t\right] & \geq \mathbb{E}_{\pi, \phi, y}\left[U\left(\pi_{\tau}, \phi_{\tau}, Y_{\tau}\right)+\int_{0}^{\tau}\left(1-\pi_{t}\right) c \alpha \phi_{t} \mathrm{~d} t\right] \\
& \geq U(\pi, \phi, y)
\end{aligned}
$$

for any stopping time $\tau$ and all $(\pi, \phi, y) \in[0,1) \times(0, \infty) \times \mathbb{R}$. By virtue of the structure of the stopping time in (3.25), it is readily seen that the inequalities in (3.39) hold with $\tau_{*}$ instead of $\tau$ when $\phi \geq h_{*}(y)$.

It remains to show that the equalities are attained in (3.39) when $\tau_{*}$ replaces $\tau$ for $(\pi, \phi, y) \in$ $[0,1) \times(0, \infty) \times \mathbb{R}$ such that $\phi<h_{*}(y)$. By virtue of the fact that the function $U(\pi, \phi, y)$ and the boundary $h_{*}(y)$ satisfy conditions (3.27) and (3.28), it follows from (3.36) and the structure of the stopping time in (3.25) that the equalities

$$
U\left(\pi_{\tau_{*} \wedge \tau_{n}}, \phi_{\tau_{*} \wedge \tau_{n}}, Y_{\tau_{*} \wedge \tau_{n}}\right)+\int_{0}^{\tau_{*} \wedge \tau_{n}}\left(1-\pi_{t}\right) c \alpha \phi_{t} \mathrm{~d} t=U(\pi, \phi, y)+M_{\tau_{*} \wedge \tau_{n}}
$$

hold for all $(\pi, \phi, y) \in[0,1) \times(0, \infty) \times \mathbb{R}$ and any localizing sequence $\left(\tau_{n}\right)_{n \in \mathbb{N}}$ of $M$. Hence, taking into account the assumption that the integral in (2.14) taken up to $\tau_{*}$ is of finite expectation and using the fact that $0 \leq U(\pi, \phi, y) \leq 1$ holds, we conclude from (3.40) that the process $\left(M_{\tau_{*} \wedge t}\right)_{t \geq 0}$ is a uniformly integrable martingale. Therefore, taking expectations in (3.40) and letting $n$ go to $\infty$, we apply the Lebesgue dominated convergence theorem to obtain the equalities

$$
\begin{aligned}
\mathbb{E}_{\pi, \phi, y}\left[1-\pi_{\tau_{*}}+\int_{0}^{\tau_{*}}\left(1-\pi_{t}\right) c \alpha \phi_{t} \mathrm{~d} t\right] & =\mathbb{E}_{\pi, \phi, y}\left[U\left(\pi_{\tau_{*}}, \phi_{\tau_{*}}, Y_{\tau_{*}}\right)+\int_{0}^{\tau_{*}}\left(1-\pi_{t}\right) c \alpha \phi_{t} \mathrm{~d} t\right] \\
& =U(\pi, \phi, y)
\end{aligned}
$$

for all $(\pi, \phi, y) \in[0,1) \times(0, \infty) \times \mathbb{R}$, which together with the inequalities in (3.39) directly imply the desired assertion.

\subsection{Main results}

We are now in a position to formulate the main assertion of the paper, which follows from a straightforward combination of Lemma 3.2 and standard change-of-variable arguments. More precisely, after obtaining the solution $U_{*}(\pi, \phi, y) \equiv(1-\pi) H_{*}(\phi, y ; z)$ with $h_{*}(y) \equiv h_{*}(y ; z)$ of the free-boundary problem (3.27)-(3.31), which satisfies conditions (3.32)-(3.34), we put $y=y(\pi, x ; z)$ and $z=x$, in order to get the solution of the initial quickest detection problem with exponential penalty costs for the detection delay stated in (2.14). 
Theorem 3.1. Suppose that the assumptions of Lemmas 3.1 and 3.2 hold. Then, in the quickest disorder detection problem (2.14) for the observation process $X$ from (2.1), the Bayesian risk function takes the form

$$
V_{*}(\pi, \phi, x)=U_{*}(\pi, \phi, y(\phi, x)) \equiv(1-\pi) H_{*}(\phi, y(\phi, x ; x) ; x)
$$

and the optimal stopping boundary $0<\lambda /(c \alpha) \leq g_{*}(x)$ in (3.13) satisfying (3.14) is uniquely determined by the equation $g(x)=h_{*}(y(g(x), x)) \equiv h_{*}(y(g(x), x ; x) ; x)$ for each $x>0$ fixed. Here, the function $U_{*}(\pi, \phi, y) \equiv(1-\pi) H_{*}(\phi, y ; z)$ and the continuous boundary of bounded variation $h_{*}(y) \equiv h_{*}(y ; z)$ form a unique solution of the free-boundary problem (3.27)-(3.34), and the expression for $y(\phi, x) \equiv y(\phi, x ; z)$ is explicitly determined by (3.23) for all $(\pi, \phi, y) \in[0,1) \times(0, \infty) \times \mathbb{R}$ and any fixed $z>0$.

Remark 3.2. Observe that the optimal stopping time in problem (2.14) does not depend on the dynamics of the process $\left(\pi_{t}\right)_{t \geq 0}$, so that the two-dimensional process $\left(\phi_{t}, X_{t}\right)_{t \geq 0}$ turns out to be a sufficient statistic. Although the process $\left(\phi_{t}, X_{t}\right)_{t \geq 0}$ is not Markovian under the probability measure $\mathbb{P}_{\pi, \phi, x}$, that property can be recognized as a consequence of the structure of the partial differential equation in (3.15)-(3.16) for the infinitesimal operator of the three-dimensional Markov process $\left(\pi_{t}, \phi_{t}, X_{t}\right)_{t \geq 0}$ and condition (3.17). This fact can also be deduced using the arguments of [1] and [2], since the quickest disorder detection problem with exponential delay penalty can be studied as an optimal stopping problem for the process $\left(\phi_{t}, X_{t}\right)_{t \geq 0}$, which becomes Markovian under an auxiliary probability measure.

Let us now give a short note concerning the case of bounded signal-to-noise ratio function $\rho(x)$ from (3.10).

Remark 3.3. Suppose that there exist some $0<\rho<\bar{\rho}<\infty$ such that $\rho \leq \rho(x) \leq \bar{\rho}$ holds for all $x>0$. Let us denote by $\underline{V}_{*}(\pi, \phi, x)$ with $\underline{g}_{*}(x)$ and by $\bar{V}_{*}(\pi, \bar{\phi}, x)$ with $\bar{g}_{*}(x)$ the solution of the Bayesian quickest disorder detection problem with exponential delay penalty, under $\rho(x) \equiv \rho$ and $\rho(x) \equiv \bar{\rho}$, respectively. In these cases, the problem of (2.14) degenerates into an optimal stopping problem for the two-dimensional Markov process $\left(\pi_{t}, \phi_{t}\right)_{t>0}$, and the value functions $\underline{V}_{*}(\pi, \phi, x) \equiv \underline{V}_{*}(\pi, \phi)$ and $\bar{V}_{*}(\pi, \phi, x) \equiv \bar{V}_{*}(\pi, \phi)$ with the stopping boundaries $\underline{g}_{*}(x) \equiv \underline{h}_{*}$ and $\bar{g}_{*}(x) \equiv \bar{h}_{*}$ are given by (3.49) and (3.48), whenever $\eta=1 / \rho$ and $\eta=1 / \bar{\rho}$, respectively. Taking into account the properties of the boundary $g_{*}(x)$ in $(3.1 \overline{4})$ and the fact that $V_{*}(\pi, \phi, x)=1-\pi$ for all $\phi \geq g_{*}(x)$ and $0 \leq \pi<1$, we therefore conclude by standard comparison arguments that the inequalities $\bar{V}_{*}(\pi, \phi) \leq V_{*}(\pi, \phi, x) \leq V_{*}(\pi, \phi)$ and, thus, $0<\lambda /(c \alpha) \leq \underline{h}_{*} \leq g_{*}(x) \leq \bar{h}_{*}$ hold for all $(\pi, \phi, x) \in[0,1) \times[0, \infty) \times(0, \infty)$.

\subsection{Some special cases}

In order to pick up some special cases in which the free-boundary problem (3.27)-(3.34) can admit a simpler structure, for the rest of the section, in addition to the conditions in (2.2), we suppose that the property

$$
\mu_{i}(x)=\frac{\eta_{i} \sigma^{2}(x)}{x} \text { for some } \eta_{i} \in \mathbb{R}, i=0,1 \text {, such that } \eta_{0} \neq \eta_{1} \text { and } \eta_{0}+\eta_{1}=1
$$

holds for all $x>0$. Moreover, we assume that the diffusion coefficient $\sigma(x)$ satisfies

$$
\sigma(x) \sim A_{0} x^{\alpha} \quad \text { as } x \downarrow 0 \quad \text { and } \quad \sigma(x) \sim A_{\infty} x^{\beta} \quad \text { as } x \uparrow \infty
$$


with some $A_{0}, A_{\infty}>0$ and $\alpha, \beta \in \mathbb{R}$ such that $(1-\alpha) \eta \leq 0$ and $(1-\beta) \eta \geq 0$ holds, where we set $\eta=1 /\left(\eta_{1}-\eta_{0}\right)$. In this case, the process $Y=\left(Y_{t}\right)_{t \geq 0}$ takes the form

$$
Y_{t}=\log \phi_{t}-\frac{1}{\eta} \log \frac{X_{t}}{z} \equiv \log \phi+\int_{0}^{t}\left(\frac{\lambda}{\phi_{s}}+\lambda+\alpha\right) \mathrm{d} s \quad \text { with } \quad \eta=\frac{1}{\eta_{1}-\eta_{0}}
$$

for any fixed $z>0$. It is easily seen from the structure of the expression in (3.43) that the one-to-one correspondence between the processes $\left(\pi_{t}, \phi_{t}, X_{t}\right)_{t \geq 0}$ and $\left(\pi_{t}, \phi_{t}, Y_{t}\right)_{t \geq 0}$ remains true in this case. Hence, getting the expression for $X_{t}$ from (3.43) and substituting it into (2.10) and (2.16), we obtain

$$
\mathrm{d} \pi_{t}=\lambda\left(1-\pi_{t}\right) \mathrm{d} t+\frac{\sigma\left(z \mathrm{e}^{-\eta Y_{t}} \phi_{t}^{\eta}\right)}{\eta z \mathrm{e}^{-\eta Y_{t}} \phi_{t}^{\eta}} \pi_{t}\left(1-\pi_{t}\right) \mathrm{d} \bar{B}_{t}
$$

with $\pi_{0}=\pi$ and

$$
\mathrm{d} \phi_{t}=\left(\lambda+(\lambda+\alpha) \phi_{t}+\frac{\sigma^{2}\left(z \mathrm{e}^{-\eta Y_{t}} \phi_{t}^{\eta}\right)}{\eta^{2} z^{2} \mathrm{e}^{-2 \eta Y_{t}} \phi_{t}^{2 \eta}} \pi_{t} \phi_{t}\right) \mathrm{d} t+\frac{\sigma\left(z \mathrm{e}^{-\eta Y_{t}} \phi_{t}^{\eta}\right)}{\eta z \mathrm{e}^{-\eta Y_{t}} \phi_{t}^{\eta}} \phi_{t} \mathrm{~d} \bar{B}_{t}
$$

with $\phi_{0}=\phi$ for any fixed $z>0$. Applying Itô's formula to (3.43) and taking into account representations (2.10) and (2.12) as well as assumption (3.41), we obtain

$$
\mathrm{d} Y_{t}=\left(\frac{\lambda}{\phi_{t}}+\lambda+\alpha\right) \mathrm{d} t
$$

with $Y_{0}=y$. It thus follows that the infinitesimal operator $\mathbb{L}_{(\pi, \phi, Y)}$ from (3.26) takes the form

$$
\begin{aligned}
\mathbb{L}_{(\pi, \phi, Y)}= & \lambda(1-\pi) \frac{\partial}{\partial \pi}+\left(\lambda+(\lambda+\alpha) \phi+\frac{\sigma^{2}\left(z \mathrm{e}^{-\eta y} \phi^{\eta}\right)}{\eta^{2} z^{2} \mathrm{e}^{-2 \eta y} \phi^{2 \eta}} \pi \phi\right) \frac{\partial}{\partial \phi}+\left(\frac{\lambda}{\phi}+\lambda+\alpha\right) \frac{\partial}{\partial y} \\
& +\frac{1}{2} \frac{\sigma^{2}\left(z \mathrm{e}^{-\eta y} \phi^{\eta}\right)}{\eta^{2} z^{2} \mathrm{e}^{-2 \eta y} \phi^{2 \eta}}\left(\pi^{2}(1-\pi)^{2} \frac{\partial^{2}}{\partial \pi^{2}}+2 \pi(1-\pi) \phi \frac{\partial^{2}}{\partial \pi \partial \phi}+\phi^{2} \frac{\partial^{2}}{\partial \phi^{2}}\right)
\end{aligned}
$$

for all $(\pi, \phi, y) \in[0,1) \times(0, \infty) \times \mathbb{R}$ and any fixed $z>0$.

\subsection{Some estimates}

Let us now introduce the function $\widehat{U}(\pi, \phi, y) \equiv(1-\pi) \widehat{H}(\phi, y)$ and the boundary $\widehat{h}(y)$ as a solution of the free-boundary problem, consisting of the differential equation

$$
\left((\lambda+(\lambda+\alpha) \phi) \frac{\partial H}{\partial \phi}+\frac{1}{2} \frac{\sigma^{2}\left(z \mathrm{e}^{-\eta y} \phi^{\eta}\right)}{\eta^{2} z^{2} \mathrm{e}^{-2 \eta y} \phi^{2 \eta}} \phi^{2} \frac{\partial^{2} H}{\partial \phi^{2}}-\lambda H\right)(\phi, y)=-c \alpha \phi \quad \text { for } \phi<h(y),
$$

instead of (3.27), for each fixed $y>0$ and conditions (3.28)-(3.30) as well as (3.32)-(3.34). The general solution of the resulting second-order ordinary differential equation in (3.45) takes the form

$$
H(\phi, y)=C_{0}(y) H_{0}(\phi, y)+C_{\infty}(y) H_{\infty}(\phi, y)-c(1+\phi),
$$

where $H_{i}(\phi, y), i=0, \infty$, form a system of fundamental positive solutions (i.e. nontrivial linearly independent particular solutions) of the corresponding homogeneous differential equation, and the $C_{i}(y), i=0, \infty$, are some arbitrary continuously differentiable functions, so that condition (3.34) holds. By virtue of the assumptions in (2.2) and taking into account 
the arguments from [11, Section 4], we can identify $H_{0}(\phi, y)$ as a decreasing solution which has a singularity at 0 and $H_{\infty}(\phi, y)$ as an increasing solution which has a singularity at $\infty$.

Observe that we should have $C_{0}(y)=0$ in (3.46), since otherwise $U(\pi, \phi, y) \equiv(1-$ $\pi) H(\phi, y) \rightarrow \pm \infty$ as $\phi \downarrow 0$, which must be excluded by virtue of the obvious fact that the value function in (3.24) is bounded at $\phi=0$ for any fixed $y \in \mathbb{R}$. Then, applying conditions (3.28) and (3.32) to the function in (3.46) with $C_{0}(y)=0$, we conclude that the equalities

$$
C_{\infty}(y) H_{\infty}(h(y), y)=c(1+h(y))+1 \quad \text { and }\left.\quad C_{\infty}(y) \frac{\partial H_{\infty}}{\partial \phi}(\phi, y)\right|_{\phi=h(y)}=c
$$

hold for fixed $y \in \mathbb{R}$. Hence, solving the equations in (3.47), we find that the solution of system (3.45) with (3.28) and (3.32)-(3.33) is given by

$$
H(\phi, y ; \widehat{h}(y))=(c(1+\widehat{h}(y))+1) \frac{H_{\infty}(\phi, y)}{H_{\infty}(\widehat{h}(y), y)}-c(1+\phi)
$$

for all $0 \leq \phi<\widehat{h}(y)$, so that $0 \leq H(\phi, y ; \widehat{h}(y)) \equiv H(\phi, y ; z ; \widehat{h}(y ; z)) \leq 1$ holds, where $\widehat{h}(y)$ satisfies the equation

$$
\left.\frac{\partial H_{\infty}}{\partial \phi}(\phi, y)\right|_{\phi=h(y)}=\frac{c H_{\infty}(h(y), y)}{c(1+h(y))+1}
$$

for any fixed $y \in \mathbb{R}$.

Taking into account the facts proved above, let us formulate the following assertion.

Corollary 3.1. Suppose that $\mu_{i}(x), i=0,1$, and $\sigma(x)>0$ are continuously differentiable functions on $(0, \infty)$ in (2.1) satisfying (2.2) and (3.41)-(3.42) with $\alpha, \beta \in \mathbb{R}$ such that $(1-\alpha) \eta \leq$ 0 and $(1-\beta) \eta \geq 0$, where $\eta=1 /\left(\eta_{1}-\eta_{0}\right)$. Assume that $\widehat{h}(y)$ provides a unique solution to (3.48) for all $y \in \mathbb{R}$. Then, using the same arguments as in the proof of Lemma 3.2 above, we can show that the function

$$
\widehat{U}(\pi, \phi, y) \equiv(1-\pi) \widehat{H}(\phi, y) \quad \text { with } \widehat{H}(\phi, y)= \begin{cases}H(\phi, y ; \widehat{h}(y)) & \text { if } 0 \leq \phi<\widehat{h}(y), \\ 1 & \text { if } \phi \geq \widehat{h}(y),\end{cases}
$$

coincides with the value function of the optimal stopping problem

$$
\begin{aligned}
\widehat{U}(\pi, \phi, y)=\inf _{\tau} \mathbb{E}_{\pi, \phi, y}\left[1-\pi_{\tau}+\int_{0}^{\tau}\left(1-\pi_{t}\right)\left(c \alpha \phi_{t}\right.\right. & -\left(\frac{\lambda}{\phi_{t}}+\lambda+\alpha\right) \frac{\partial \widehat{H}}{\partial y}\left(\phi_{t}, Y_{t}\right) \\
& \left.\left.\times \mathbf{1}\left(\phi_{t}<\widehat{h}\left(Y_{t}\right)\right)\right) \mathrm{d} t\right],
\end{aligned}
$$

which corresponds to the Bayesian risk function in (3.24). Moreover, $\widehat{h}(y) \equiv \widehat{h}(y ; z)$ determined by (3.48) provides a hitting boundary for the stopping time

$$
\widehat{\tau}=\inf \left\{t \geq 0 \mid \phi_{t} \geq \widehat{h}\left(Y_{t}\right)\right\}
$$

which turns out to be optimal in (3.50) whenever the integral above is of finite expectation, and $\widehat{\tau}=0$ otherwise, for any fixed $z>0$.

Remark 3.4. Note that the function $\widehat{U}(\pi, \phi, y)$ in (3.50) and the boundary $\widehat{h}(y)$ in $(3.51)$ provide lower (upper) and upper (lower) estimates for the initial value function $U_{*}(\pi, \phi, y)$ in 
(3.24) and the optimal stopping boundary $h_{*}(y)$ in (3.25), whenever the function $y \mapsto \widehat{H}(\phi, y)$ is increasing (decreasing) on $\mathbb{R}$. According to Lemma 3.1 and the structure of the change of variables in (3.23), such a situation occurs when $\rho(x)$ from (3.10) is an increasing (decreasing) function on $(0, \infty)$ and $\eta_{0}<\eta_{1}\left(\eta_{0}>\eta_{1}\right)$ in (3.41), respectively.

\section{The case of linear delay penalty costs}

In this section we provide some results related to the quickest detection problem with linear delay penalty costs of Example 2.1.

\subsection{The structure of the continuation region}

Following the arguments of Subsection 3.1 and applying Doob's optional sampling theorem, it follows from (3.2) that the equality

$$
\mathbb{E}_{\pi, \varphi, x}\left[1-\pi_{\tau}+\int_{0}^{\tau}\left(1-\pi_{t}\right) c \varphi_{t} \mathrm{~d} t\right]=1-\pi+\mathbb{E}_{\pi, \varphi, x} \int_{0}^{\tau}\left(1-\pi_{t}\right)\left(c \varphi_{t}-\lambda\right) \mathrm{d} t
$$

holds for all $(\pi, \varphi, x) \in[0,1) \times[0, \infty) \times(0, \infty)$ and any stopping time $\tau$ satisfying $\mathbb{E}_{\pi, \varphi, x}<\infty$. Taking into account the structure of the reward in (2.13), it is also seen from (4.1) that it is never optimal to stop when $\varphi_{t}<\lambda / c$ for any $t \geq 0$. This shows that all the points $(\pi, \varphi, x)$ such that $\varphi<\lambda / c$ belong to the continuation region

$$
C^{\prime}=\left\{(\pi, \varphi, x) \in[0,1) \times[0, \infty) \times(0, \infty) \mid V^{\prime}(\pi, \varphi, x)<1-\pi\right\} .
$$

Then, combining the arguments in [28, Chapter IV, Section 3] with those in Subsection 3.2, we conclude that the continuation region in (4.2) for the optimal stopping problem (2.13) takes the form

$$
C^{\prime}=\left\{(\pi, \varphi, x) \in[0,1) \times[0, \infty) \times(0, \infty) \mid \varphi<g^{\prime}(x)\right\},
$$

so that the corresponding stopping region is the closure of the set

$$
D^{\prime}=\left\{(\pi, \varphi, x) \in[0,1) \times[0, \infty) \times(0, \infty) \mid \varphi>g^{\prime}(x)\right\} .
$$

\subsection{The optimal stopping boundary}

In order to characterize the behavior of the boundary $g^{\prime}(x)$ in (4.3)-(4.4), we apply the integration-by-parts formula to the processes from (2.9) and (3.2) to conclude that the expression

$$
\int_{0}^{\tau^{\prime}}\left(1-\pi_{t}\right)\left(c \varphi_{t}-\lambda\right) \mathrm{d} t=\int_{0}^{\tau^{\prime}}\left((1-\pi)\left(\frac{c \pi}{1-\pi}-\lambda\right)+\int_{0}^{t} \lambda(c+\lambda)\left(1-\pi_{s}\right) \mathrm{d} s+N_{t}^{\prime}\right) \mathrm{d} t
$$

holds for the optimal stopping time $\tau^{\prime}=\tau^{\prime}(\pi, \varphi, x)$ in (2.13) such that $(\pi, \varphi, x) \in C^{\prime}$. Here, the process $N^{\prime}=\left(N_{t}^{\prime}\right)_{t \geq 0}$ defined by

$$
N_{t}^{\prime}=\int_{0}^{t} \frac{\mu_{1}\left(X_{s}\right)-\mu_{0}\left(X_{s}\right)}{\sigma\left(X_{s}\right)}(c+\lambda) \pi_{s}\left(1-\pi_{s}\right) \mathrm{d} \bar{B}_{s}
$$

is a continuous local martingale under $\mathbb{P}_{\pi, \varphi, x}$, and its quadratic variation process is given by

$$
\left\langle N^{\prime}\right\rangle_{t}=\int_{0}^{t}\left(\frac{\mu_{1}\left(X_{s}\right)-\mu_{0}\left(X_{s}\right)}{\sigma\left(X_{s}\right)}\right)^{2}(c+\lambda)^{2} \pi_{s}^{2}\left(1-\pi_{s}\right)^{2} \mathrm{~d} s .
$$


Let us now take $x^{\prime}>0$ such that $x<x^{\prime}$. Then, by means of the arguments from the previous section, we conclude that the process $N^{\prime}$ has a larger quadratic variation when the process $X$ starts at $x^{\prime}$ rather than at $x$, whenever the function $\rho(x)$ from $(3.10)$ is increasing on $(0, \infty)$. Suppose that the process $\left(N_{\tau^{\prime} \wedge t}^{\prime}\right)_{t \geq 0}$ is a uniformly integrable martingale under both $\mathbb{P}_{\pi, \varphi, x}$ and $\mathbb{P}_{\pi, \varphi, x^{\prime}}$, which is the case for the process $\left(M_{\tau_{*} \wedge t}\right)_{t \geq 0}$ from (3.37) under the conditions of Lemma 3.2. Then, it follows from the assumption that the integral in (2.13) taken up to the optimal stopping time $\tau^{\prime}$ is of finite expectation and the third inequality in (2.2) that the integrals on the both sides of the expression in (4.5) are of finite expectation too. Hence, taking into account the fact that $\tau^{\prime}$ has the structure of the first time the process $\left(\varphi_{t}\right)_{t \geq 0}$ hits an upper boundary, by virtue of arguments similar to those used in the case of constant coefficients $\mu_{i}, i=0,1$, and $\sigma>0$ in (2.1), we conclude that the inequality

$$
\begin{aligned}
\mathbb{E}_{\pi, \varphi, x^{\prime}} & \int_{0}^{\tau^{\prime}}\left((1-\pi)\left(\frac{c \pi}{1-\pi}-\lambda\right)+\int_{0}^{t} \lambda(c+\lambda)\left(1-\pi_{s}\right) \mathrm{d} s+N_{t}^{\prime}\right) \mathrm{d} t \\
\leq & \mathbb{E}_{\pi, \varphi, x} \int_{0}^{\tau^{\prime}}\left((1-\pi)\left(\frac{c \pi}{1-\pi}-\lambda\right)+\int_{0}^{t} \lambda(c+\lambda)\left(1-\pi_{s}\right) \mathrm{d} s+N_{t}^{\prime}\right) \mathrm{d} t
\end{aligned}
$$

is satisfied, whenever $\rho(x)$ is increasing on $(0, \infty)$. Finally, gathering expressions (4.1), (4.5), and (4.6), we see that

$$
\begin{aligned}
V^{\prime}\left(\pi, \varphi, x^{\prime}\right)-(1-\pi) & \leq \mathbb{E}_{\pi, \varphi, x^{\prime}} \int_{0}^{\tau^{\prime}}\left(1-\pi_{t}\right)\left(c \varphi_{t}-\lambda\right) \mathrm{d} t \\
& \leq \mathbb{E}_{\pi, \varphi, x} \int_{0}^{\tau^{\prime}}\left(1-\pi_{t}\right)\left(c \varphi_{t}-\lambda\right) \mathrm{d} t \\
& =V^{\prime}(\pi, \varphi, x)-(1-\pi)
\end{aligned}
$$

holds, whenever $\rho(x)$ is increasing on $(0, \infty)$. By virtue of inequality (4.7), we may therefore conclude that $\left(\pi, \varphi, x^{\prime}\right) \in C^{\prime}$, so that the boundary $g^{\prime}(x)$ is increasing or decreasing in (4.3)(4.4), whenever $\rho(x)$ is increasing or decreasing on $(0, \infty)$, respectively.

Summarizing the facts proved above, we now formulate the assertions related to the Bayesian quickest detection problem with linear penalty costs for a detection delay, which are proved using arguments from the previous section.

Lemma 4.1. Suppose that $\mu_{i}(x), i=0,1$, and $\sigma(x)>0$ are continuously differentiable functions on $(0, \infty)$ in (2.1) satisfying (2.2). Then the optimal Bayesian time of alarm $\tau^{\prime}$ in the quickest disorder detection problem (2.13) has the structure

$$
\tau^{\prime}=\inf \left\{t \geq 0 \mid \varphi_{t} \geq g^{\prime}\left(X_{t}\right)\right\}
$$

whenever $\mathbb{E}_{\pi, \varphi, x} \tau^{\prime}<\infty$ holds for all $(\pi, \varphi, x) \in[0,1) \times[0, \infty) \times(0, \infty)$, and $\tau^{\prime}=0$ otherwise. Moreover, the property

$$
g^{\prime}(x):(0, \infty) \rightarrow\left(\frac{\lambda}{c}, \infty\right) \text { is increasing/decreasing if } \rho(x) \text { is increasing/decreasing }
$$

holds with $\rho(x)$ defined in (3.10) for all $x>0$.

Theorem 4.1. Suppose that the assumptions of Lemmas 4.1 and 3.2 hold with $\phi=\varphi, \alpha=0$ in (3.26), and $\alpha=1$ in (3.27). Then, in the quickest disorder detection problem (2.13) for the 
observation process $X$ from (2.1), the Bayesian risk function takes the form

$$
V^{\prime}(\pi, \varphi, x)=U^{\prime}(\pi, \varphi, y(\varphi, x)) \equiv(1-\pi) H^{\prime}(\varphi, y(\varphi, x ; x) ; x)
$$

and the optimal stopping boundary $0<\lambda / c \leq g^{\prime}(x)$ in (4.8) satisfying (4.9) is uniquely determined by the equation $g(x)=h^{\prime}(y(g(x), x)) \equiv h^{\prime}(y(g(x), x ; x) ; x)$ for each fixed $x>0$. Here, the function $U^{\prime}(\pi, \varphi, y) \equiv(1-\pi) H^{\prime}(\varphi, y ; z)$ and the bounded continuous boundary of bounded variation $h^{\prime}(y) \equiv h^{\prime}(y ; z)$ form a unique solution of the free-boundary problem (3.27)-(3.34), and the expression for $y(\varphi, x) \equiv y(\varphi, x ; z)$ is explicitly determined by relation (3.23) with $\phi=\varphi$ for all $(\pi, \varphi, y) \in[0,1) \times(0, \infty) \times \mathbb{R}$ and any $z>0$ fixed.

Remark 4.1. Suppose that there exist some $0<\underline{\rho}<\bar{\rho}<\infty$ such that $\rho \leq \rho(x) \leq \bar{\rho}$ holds for all $x>0$. Let us denote by $\underline{V}^{\prime}(\pi, \varphi, x)$ with $g^{\prime}(x)$ and by $\bar{V}^{\prime}(\pi, \varphi, x)$ with $\bar{g}^{\prime}(x)$ the solution of the Bayesian quickest disorder detection problem with linear delay penalty, under $\rho(x) \equiv \underline{\rho}$ and $\rho(x) \equiv \bar{\rho}$, respectively. In these cases, problem (2.13) degenerates into an optimal stopping problem for the one-dimensional Markov process $\left(\pi_{t}\right)_{t \geq 0}$ being equivalent to $\left(\varphi_{t}\right)_{t \geq 0}$, and the value functions $\underline{V}^{\prime}(\pi, \varphi, x) \equiv \underline{V}^{\prime}(\pi, \varphi) \equiv \underline{V}^{\prime}(\varphi /(1+\varphi), \varphi)$ and $\bar{V}^{\prime}(\pi, \varphi, x) \equiv \bar{V}^{\prime}(\pi, \varphi) \equiv \bar{V}^{\prime}(\varphi /(1+\varphi), \varphi)$ with the stopping boundaries $\underline{g}^{\prime}(x) \equiv \underline{h}^{\prime}$ and $\bar{g}^{\prime}(x) \equiv \bar{h}^{\prime}$ are given by (4.13) and (4.12) below, whenever $\eta=1 / \rho$ and $\bar{\eta}=1 / \bar{\rho}$, respectively. Taking into account the properties of the boundary $g^{\prime}(x)$ in (4.9) and the fact that $V^{\prime}(\pi, \varphi, x)=1-\pi$ for all $\varphi \geq g^{\prime}(x)$ and $0 \leq \pi<1$, we therefore conclude by standard comparison arguments that the inequalities $\bar{V}^{\prime}(\pi, \varphi) \leq V^{\prime}(\pi, \varphi, x) \leq \underline{V^{\prime}}(\pi, \varphi)$ and, thus, $0<\lambda / c \leq \underline{h}^{\prime} \leq g^{\prime}(x) \leq \bar{h}^{\prime}$ hold for all $(\pi, \varphi, x) \in[0,1) \times[0, \infty) \times(0, \infty)$.

\subsection{Some estimates}

Let us finally introduce the function $\widetilde{U}(\varphi /(1+\varphi), \varphi, y) \equiv \widetilde{G}(\varphi, y)$ and the boundary $\widehat{h}(y)$ as a solution of the free-boundary problem consisting of the differential equation

$$
\begin{aligned}
\left(\lambda(1+\varphi) \frac{\partial G}{\partial \varphi}+\frac{\sigma^{2}\left(z \mathrm{e}^{-\eta y} \varphi^{\eta}\right)}{\eta^{2} z^{2} \mathrm{e}^{-2 \eta y} \varphi^{2 \eta}}\left(\frac{\varphi^{2}}{1+\varphi} \frac{\partial G}{\partial \varphi}+\frac{\varphi^{2}}{2} \frac{\partial^{2} G}{\partial \varphi^{2}}\right)\right)(\varphi, y) \\
=-\frac{c \varphi}{1+\varphi} \text { for } \varphi<h(y),
\end{aligned}
$$

instead of (3.27), for each fixed $y>0$ and conditions (3.28)-(3.30) as well as (3.32)-(3.34) with $\phi=\varphi$ and $\pi=\varphi /(1+\varphi)$. The general solution of the resulting first-order linear ordinary differential equation for $\varphi \mapsto(\partial G / \partial \varphi)(\varphi, y)$ takes the form

$$
\begin{aligned}
\frac{\partial G}{\partial \varphi}(\varphi, y)= & \frac{C(y)}{(1+\varphi)^{2}} \exp \left(\int_{\varphi}^{w} \frac{\lambda(1+u)}{u^{2}} \frac{2 \eta^{2} z^{2} \mathrm{e}^{-2 \eta y} u^{2 \eta}}{\sigma^{2}\left(z \mathrm{e}^{-\eta y} u^{\eta}\right)} \mathrm{d} u\right) \\
& -\int_{0}^{\varphi} \frac{c(1+u)}{u(1+\varphi)^{2}} \frac{2 \eta^{2} z^{2} \mathrm{e}^{-2 \eta y} u^{2 \eta}}{\sigma^{2}\left(z \mathrm{e}^{-\eta y} u^{\eta}\right)} \exp \left(-\int_{u}^{\varphi} \frac{\lambda(1+v)}{v^{2}} \frac{2 \eta^{2} z^{2} \mathrm{e}^{-2 \eta y} v^{2 \eta}}{\sigma^{2}\left(z \mathrm{e}^{-\eta y} v^{\eta}\right)} \mathrm{d} v\right) \mathrm{d} u,
\end{aligned}
$$

where $C(y)$ is an arbitrary continuously differentiable function for each $y \in \mathbb{R}$ and any fixed $z, w>0$. By virtue of the assumptions in (2.2), we see that the term in the first line of (4.11) above tends to $\infty$ as $\varphi \downarrow 0$, so that $(\partial G / \partial \varphi)(\varphi, y) \rightarrow \pm \infty$ as $C(y) \neq 0$ for any fixed $y \in \mathbb{R}$. We should thus choose $C(y)=0$, which is equivalent to the property in (3.33). Hence, integrating (4.11), we therefore find that the solution of system (4.10) with (3.28) and (3.32)-(3.33) is 
given by

$$
\begin{aligned}
& G(\varphi, y ; \tilde{h}(y))= \frac{1}{1+\widetilde{h}(y)} \\
&+\int_{\varphi}^{\tilde{h}(y)} \int_{0}^{w} \frac{c(1+u)}{u(1+w)^{2}} \frac{2 \eta^{2} z^{2} \mathrm{e}^{-2 \eta y} u^{2 \eta}}{\sigma^{2}\left(z \mathrm{e}^{-\eta y} u^{\eta}\right)} \\
& \quad \quad \times \exp \left(-\int_{u}^{w} \frac{\lambda(1+v)}{v^{2}} \frac{2 \eta^{2} z^{2} \mathrm{e}^{-2 \eta y} v^{2 \eta}}{\sigma^{2}\left(z \mathrm{e}^{-\eta y} v^{\eta}\right)} \mathrm{d} v\right) \mathrm{d} u \mathrm{~d} w
\end{aligned}
$$

for all $0 \leq \varphi<\widetilde{h}(y)$, so that $0 \leq G(\varphi, y ; \widetilde{h}(y)) \equiv G(\varphi, y ; z ; \tilde{h}(y ; z)) \leq 1 /(1+\varphi)$ holds, where $\tilde{h}(y)$ satisfies the equation

$$
\int_{0}^{h(y)} \frac{c(1+u)}{u} \frac{2 \eta^{2} z^{2} \mathrm{e}^{-2 \eta y} u^{2 \eta}}{\sigma^{2}\left(z \mathrm{e}^{-\eta y} u^{\eta}\right)} \exp \left(-\int_{u}^{h(y)} \frac{\lambda(1+v)}{v^{2}} \frac{2 \eta^{2} z^{2} \mathrm{e}^{-2 \eta y} v^{2 \eta}}{\sigma^{2}\left(z \mathrm{e}^{-\eta y} v^{\eta}\right)} \mathrm{d} v\right) \mathrm{d} u=1
$$

for each $y \in \mathbb{R}$ and any fixed $z>0$.

Summarizing the above facts, let us formulate the following assertion.

Corollary 4.1. Suppose that $\mu_{i}(x), i=0,1$, and $\sigma(x)>0$ are continuously differentiable functions on $(0, \infty)$ in (2.1) satisfying (2.2) and (3.41)-(3.42) with $\alpha, \beta \in \mathbb{R}$ such that $(1-\alpha) \eta \leq 0$ and $(1-\beta) \eta \geq 0$, where $\eta=1 /\left(\eta_{1}-\eta_{0}\right)$. Assume that $\tilde{h}(y)$ provides a unique solution to (4.12) for all $y \in \mathbb{R}$. Then, using the same arguments as in the proof of Lemma 3.2, we show that the function

$$
\widetilde{U}(\pi, \varphi, y) \equiv \widetilde{G}(\varphi, y)= \begin{cases}G(\varphi, y ; \tilde{h}(y)) & \text { if } 0 \leq \varphi<\tilde{h}(y), \\ \frac{1}{1+\varphi} & \text { if } \varphi \geq \widetilde{h}(y),\end{cases}
$$

coincides with the value function of the optimal stopping problem

$$
\begin{aligned}
\widetilde{U}(\pi, \varphi, y)=\inf _{\tau} \mathbb{E}_{\pi, \varphi, y}\left[\frac{1}{1+\varphi_{\tau}}+\int_{0}^{\tau}\left(\frac{c \varphi_{t}}{1+\varphi_{t}}-\right.\right. & \left(\frac{\lambda}{\varphi_{t}}+\lambda\right) \frac{\partial \widetilde{G}}{\partial y}\left(\varphi_{t}, Y_{t}\right) \\
& \left.\left.\times \mathbf{1}\left(\varphi_{t}<\tilde{h}\left(Y_{t}\right)\right)\right) \mathrm{d} t\right]
\end{aligned}
$$

with $\pi=\varphi /(1+\varphi)$, which corresponds to the Bayesian risk function in (2.13). Moreover, $0<\lambda / c \leq \widetilde{h}(y) \equiv \widetilde{h}(y ; z)$ determined by (4.12) provides a hitting boundary for the stopping time

$$
\tilde{\tau}=\inf \left\{t \geq 0 \mid \varphi_{t} \geq \tilde{h}\left(Y_{t}\right)\right\},
$$

which turns out to be optimal in (4.14) whenever the integral above is of finite expectation for any fixed $z>0$.

Remark 4.2. Note that the function $\widetilde{U}(\pi, \varphi, y)$ in (4.14) and the boundary $\widetilde{h}(y)$ in (4.15) provide lower (upper) and upper (lower) estimates for the initial value function $U^{\prime}(\pi, \varphi, y)$ defined as in (3.24) with $\alpha=1$ and the optimal stopping boundary $h^{\prime}(y)$ defined as in (3.25) with $\phi=\varphi$, whenever the function $y \mapsto \widetilde{G}(\varphi, y)$ is increasing (decreasing) on $\mathbb{R}$. According to Lemma 4.1 and the structure of the change of variables in (3.23) with $\phi=\varphi$, such a situation occurs when $\rho(x)$ from (3.10) is an increasing (decreasing) function on $(0, \infty)$ and $\eta_{0}<\eta_{1}$ $\left(\eta_{0}>\eta_{1}\right)$ in $(3.41)$, respectively. 


\section{Acknowledgements}

The authors are grateful to the anonymous referees for their careful reading of the paper and helpful comments. The paper was written when the first author was visiting the Department of Mathematical Stochastics at the University of Freiburg in Germany in the academic year 2011/2012. The hospitality and financial support from the Alexander von Humboldt Foundation through the fellowship for experienced researchers is gratefully acknowledged.

\section{References}

[1] Bayraktar, E. and Dayanik, S. (2006). Poisson disorder problem with exponential penalty for delay. Math. Operat. Res. 31, 217-233.

[2] Bayraktar, E., Dayanik, S. And Karatzas, I. (2005). The standard Poisson disorder problem revisited. Stoch. Process. Appl. 115, 1437-1450.

[3] Bayraktar, E., Dayanik, S. and Karatzas, I. (2006). Adaptive Poisson disorder problem. Ann. Appl. Prob. 16, 1190-1261.

[4] Beibel, M. (2000). A note on sequential detection with exponential penalty for the delay. Ann. Statist. 28, 1696-1701.

[5] Bensoussan, A. And Lions, J.-L. (1982). Applications of Variational Inequalities in Stochastic Control. NorthHolland, Amsterdam.

[6] Dayanik, S. (2010). Compound Poisson disorder problems with nonlinear detection delay penalty cost functions. Sequent. Anal. 29, 193-216.

[7] Dayanik, S. and Sezer, S. O. (2006). Compound Poisson disorder problem. Math. Operat. Res. 31, 649-672.

[8] Dynkin, E. B. (1963). The optimum choice of the instant for stopping a Markov process. Soviet Math. Dokl. 4, 627-629.

[9] Friedman, A. (1976). Stochastic Differential Equations and Applications, Vol. 2. Academic Press, New York.

[10] Gapeev, P. V. and Peskir, G. (2006). The Wiener disorder problem with finite horizon. Stoch. Process. Appl. 116, 1770-1791.

[11] Gapeev, P. V. and Shiryaev, A. N. (2011). On the sequential testing problem for some diffusion processes. Stochastics 83, 519-535.

[12] Grigelionis, B. I. And Shiryaev, A. N. (1966). On Stefan's problem and optimal stopping rules for Markov processes. Theory Prob. Appl. 11, 541-558.

[13] Kolmogorov, A. N. (1992). On analytical methods in probability theory. In Selected Works of A. N. Kolmogorov, vol. II, ed. A. N. Shiryaev, Kluwer, Dordrecht, pp. 62-108.

[14] Krylov, N. V. (1980). Controlled Diffusion Processes. Springer, New York.

[15] Liptser, R. S. And ShiryaeV, A. N. (1977). Statistics of Random Processes I. Springer, Berlin.

[16] ØKSEndal, B. (1998). Stochastic Differential Equations. Springer, Berlin.

[17] Peskir, G. (2007). A change-of-variable formula with local time on surfaces. In Séminaire de Probababilité XL (Lecture Notes Math. 1899). Springer, Berlin, pp. 69-96.

[18] Peskir, G. And Shiryaev, A. N. (2006). Optimal Stopping and Free-Boundary Problems. Birkhäuser, Basel.

[19] Poor, H. V. (1998). Quickest detection with exponential penalty for delay. Ann. Statist. 26, 2179-2205.

[20] Poor, H. V. ANd HadjILIAdis, O. (2008). Quickest Detection. Cambridge University Press.

[21] Pospisil, L., Vecer, J. and Hadjiliadis, O. (2009). Formulas for stopped diffusion processes with stopping times based on drawdowns and drawups. Stochastic Process. Appl. 119, 2563-2578.

[22] Revuz, D. AND Yor, M. (1999). Continuous Martingales and Brownian Motion. Springer, Berlin.

[23] Shiryaev, A. N. (1961). The problem of the most rapid detection of a disturbance in a stationary process. Soviet Math. Dokl. 2, 795-799.

[24] Shiryaev, A. N. (1963). On optimum methods in quickest detection problems. Theory Prob. Appl. 8, 22-46.

[25] Shiryaev, A. N. (1964). On Markov sufficient statistics in nonadditive Bayes problems of sequential analysis. Theory Prob. Appl. 9, 670-686.

[26] Shiryaev, A. N. (1965). Some exact formulas in a 'disorder' problem. Theory Prob. Appl. 10, 348-354.

[27] Shiryaev, A. N. (1967). Two problems of sequential analysis. Cybernetics 3, 63-69.

[28] Shiryaev, A. N. (1978). Optimal Stopping Rules. Springer, Berlin.

[29] Shiryaev, A. N. (2002). Quickest detection problems in the technical analysis of the financial data. In Mathematical Finance-Bachelier Congress 2000 (Paris, June/July 2000), eds. H. Geman et al., Springer, Berlin, pp. 487-521. 
[30] Shiryaev, A. N. (2008). Generalized Bayesian nonlinear quickest detection problems: on Markov family of sufficient statistics. In Mathematical Control Theory and Finance (Lisbon, April 2007), eds. A. Sarychev et al., Springer, Berlin, pp. 377-386.

[31] Shiryaev, A. N. AND Zryumov, P. Y. (2009). On the linear and nonlinear generalized Bayesian disorder problem (discrete time case). In Optimality and Risk-Modern Trends in Mathematical Finance, eds. F. Delbaen et al., Springer, Berlin, pp. 227-235.

[32] Veretennikov, A. Yu. (1980). On the strong solutions of stochastic differential equations. Theory Prob. Appl. 24, 354-366. 